\title{
Bureau d'expertises extrajudiciaires de la FMH: rapport annuel 2012
}

\section{Valérie Rothhardt}

Avocate, service juridique de la FMH
1 Pour faciliter la lecture, la forme masculine est utilisée dans ce texte pour désigner des personnes, mais elle englobe toujours les personnes des deux sexes.
Le Bureau d'expertises extrajudiciaires de la FMH a pour but de mandater un ou plusieurs experts ${ }^{1}$, à la demande d'un patient ayant été traité en Suisse, pour déterminer si, dans le cas concret, un médecin exerçant en pratique privée ou à l'hôpital a commis une faute de diagnostic ou de traitement. Les experts sont proposés par la société de discipline médicale concernée, ce qui permet de trouver des experts indépendants et compétents. Ce sont les assurances de responsabilité civile (ci-après assurances RC) des médecins qui prennent en charge les honoraires des experts, le patient devant uniquement s'acquitter d'une taxe administrative de 600 francs plus TVA.

Ainsi, le Bureau d'expertises est un instrument utile et efficace pour les patients et pour les médecins. En effet, d'un côté, il permet aux patients de faire éclaircir la question de savoir s'ils ont été victimes d'une faute médicale à des coûts peu élevés. De l'autre côté, il donne une base fiable aux médecins, respectivement à leur assurance RC, qui leur permettra de régler au mieux le litige.

En 2012, le Bureau d'expertises extrajudiciaires de la FMH a procédé à l'établissement de 64 expertises. Les experts ont conclu à une ou plusieurs fautes de diagnostic ou de traitement dans 30 cas et n'ont constaté aucune faute dans 32 autres cas.

Le Bureau d'expertises de la FMH n'est pas compétent pour tous les litiges. Pour qu'il organise une

\section{Statistiques du Bureau d'expertises extrajudiciaires 2012}

\section{Méthode de classification}

En cas d'expertise multidisciplinaire, la classification s'effectue selon la discipline la plus touchée par le cas. Exemple: s'il est fait appel à une équipe d'experts principalement en gynécologie et secondairement en anesthésiologie et qu'une faute est reconnue seulement en gynécologie, l'expertise sera classée dans la catégorie «gynécologie, faute constatée». Si, dans le même cas, une faute est reconnue en anesthésiologie, et non pas en gynécologie, l'expertise sera classée exclusivement dans la catégorie «anesthésiologie, faute constatée». Si une faute est reconnue dans ces deux disciplines, l'expertise apparait dans la statistique sous «gynécologie, faute constatée».

La statistique reflète ainsi le résultat déterminant pour le patient et non pas la mesure du travail total fourni par les experts.

\section{Analyse de la statistique et limites quant à son interprétation}

En 2012, 64 expertises ont été menées à bien alors que leur nombre s'était élevé à 77 durant l'exercice précédent. Dans un peu plus d'un tiers (37\%) des cas examinés, il s'est agi d'analyser exclusivement des traitements prodigués par des médecins en cabinet

\section{«Les experts sont proposés par la société de discipline médicale concer- née, ce qui permet de trouver des experts indépendants et compétents.»}

expertise, il faut notamment que le patient ait subi une atteinte considérable à sa santé et qu'il n'ait pas pu trouver d'accord sans expertise avec l'assureur RC du médecin ou de l'hôpital. Il faut également qu'aucun tribunal n'ait été saisi du litige ni n'ait prononcé de jugement à ce sujet.

La demande, que le patient doit motiver de manière détaillée, permet de bien saisir quelle/s société/s de discipline médicale est/sont concernée/s et de déterminer la complexité du cas. Le Bureau d'expertises extrajudiciaires de la FMH peut ainsi octroyer son mandat d'expertise à l'équipe d'experts adéquate pour examiner le cas litigieux. Dans bien des situations, l'équipe d'experts doit se composer de représentants de plusieurs disciplines médicales. privé. Quant au reste des cas (moins des deux tiers), ils ont porté soit exclusivement sur l'analyse de traitements hospitaliers, soit sur des traitements impliquant conjointement des cabinets privés et des hôpitaux. Les expertises réalisées en 2012 ont nécessité l'intervention de 21 équipes multidisciplinaires d'experts.

Le pourcentage de fautes reconnues a légèrement augmenté par rapport à l'année précédente et s'élève à 46,9\% pour 2012 (contre $44,2 \%$ en 2011).

Ces 10 dernières années, soit entre 2003 et 2012, le pourcentage de fautes reconnues a été compris entre $34,9 \%$ (en 2004) et 50,6\% (en 2010). Le pourcentage de fautes niées s'est élevé entre $45,7 \%$ et $65,1 \%$. 


\begin{tabular}{|c|c|c|c|c|c|}
\hline \multicolumn{6}{|l|}{ Tableau 1} \\
\hline \multicolumn{6}{|l|}{ Vue d'ensemble 1982-2012. } \\
\hline & Période & $\begin{array}{l}\text { Expertises } \\
\text { établies }\end{array}$ & $\begin{array}{l}\text { Faute/s de traite- } \\
\text { ment ou de dia- } \\
\text { gnostic avérée/s }\end{array}$ & $\begin{array}{l}\text { Faute/s de traite- } \\
\text { ment ou de dia- } \\
\text { gnostic niée/s }\end{array}$ & $\begin{array}{l}\text { Faute/s de traitement } \\
\text { ou de diagnostic } \\
\text { indéterminée/s }\end{array}$ \\
\hline Toute la Suisse & 1982-2011 & 3391 & 1140 & 2155 & 96 \\
\hline Suisse alémanique et Tessin & 2012 & 36 & 18 & 17 & 1 \\
\hline Romandie & 2012 & 28 & 12 & 15 & 1 \\
\hline \multirow[t]{2}{*}{ Toute la Suisse } & 2012 & 64 & 30 & 32 & 2 \\
\hline & & $100 \%$ & $46,9 \%$ & $50,0 \%$ & $3,1 \%$ \\
\hline \multirow[t]{2}{*}{ Toute la Suisse } & 1982-2012 & 3455 & 1170 & 2187 & 98 \\
\hline & & $100 \%$ & $33,9 \%$ & $63,3 \%$ & $2,8 \%$ \\
\hline $\begin{array}{l}\text { Toute la Suisse } \\
\text { (10 dernières années) }\end{array}$ & $\begin{array}{l}2002- \\
2012\end{array}$ & $\begin{array}{l}804 \\
100 \%\end{array}$ & $\begin{array}{l}357 \\
44,4 \%\end{array}$ & $\begin{array}{l}432 \\
53,7 \%\end{array}$ & $\begin{array}{l}15 \\
1,9 \%\end{array}$ \\
\hline
\end{tabular}

\section{Tableau 2}

Résultats par spécialité 1982-2012.

\begin{tabular}{|c|c|c|c|c|}
\hline & $\begin{array}{l}\text { Expertises } \\
\text { établies }\end{array}$ & $\begin{array}{l}\text { Faute/s de traitement } \\
\text { ou de diagnostic } \\
\text { avérée/s }\end{array}$ & $\begin{array}{l}\text { Faute/s de traite- } \\
\text { ment ou de } \\
\text { diagnostic niée/s }\end{array}$ & $\begin{array}{l}\text { Faute/s de traitement } \\
\text { ou de diagnostic } \\
\text { indéterminée/s }\end{array}$ \\
\hline Médecine générale & 239 & 88 & 141 & 10 \\
\hline Anesthésiologie & 118 & 38 & 77 & 3 \\
\hline Chirurgie & 830 & 293 & 510 & 27 \\
\hline Dermatologie & 30 & 9 & 19 & 2 \\
\hline Gastro-entérologie & 14 & 2 & 12 & 0 \\
\hline Gynécologie et obstétrique & 438 & 166 & 264 & 8 \\
\hline Chirurgie de la main & 52 & 19 & 31 & 2 \\
\hline $\begin{array}{l}\text { Chirurgie cardiaque } \\
\text { et vasculaire thoracique }\end{array}$ & 25 & 8 & 16 & 1 \\
\hline Médecine interne & 233 & 78 & 151 & 4 \\
\hline Cardiologie & 22 & 12 & 9 & 1 \\
\hline Chirurgie maxillo-faciale & 23 & 3 & 20 & 0 \\
\hline Chirurgie pédiatrique & 14 & 4 & 10 & 0 \\
\hline Psychiatrie pédiatrique & 1 & 0 & 1 & 0 \\
\hline Néphrologie & 2 & 0 & 2 & 0 \\
\hline Neurochirurgie & 92 & 25 & 65 & 2 \\
\hline Neurologie & 25 & 7 & 17 & 1 \\
\hline Oncologie & 9 & 4 & 5 & 0 \\
\hline Ophtalmologie & 133 & 40 & 87 & 6 \\
\hline Chirurgie orthopédique & 657 & 243 & 399 & 15 \\
\hline Oto-rhino-laryngologie ORL & 120 & 27 & 89 & 4 \\
\hline Pédiatrie & 68 & 28 & 37 & 3 \\
\hline Pathologie & 6 & 4 & 2 & 0 \\
\hline Pharmacologie & 2 & 2 & 0 & 0 \\
\hline $\begin{array}{l}\text { Médecine physique } \\
\text { et réadaptation }\end{array}$ & 13 & 3 & 9 & 1 \\
\hline $\begin{array}{l}\text { Chirurgie plastique, reconstruc- } \\
\text { tive et esthétique }\end{array}$ & 128 & 27 & 99 & 2 \\
\hline Pneumologie & 2 & 1 & 1 & 0 \\
\hline Psychiatrie & 15 & 7 & 8 & 0 \\
\hline Radiologie & 51 & 14 & 34 & 3 \\
\hline Radio-oncologie & 1 & 1 & 0 & 0 \\
\hline Rhumatologie & 16 & 5 & 11 & 0 \\
\hline Urologie & 76 & 12 & 61 & 3 \\
\hline Total 1982-2012 & 3455 & 1170 & 2187 & 98 \\
\hline
\end{tabular}


Ces 5 dernières années, soit entre 2008 et 2012, le pourcentage de fautes reconnues a oscillé entre $44,2 \%$ et $50,6 \%$. Quant au pourcentage de fautes niées pour cette même période, il a varié entre $45,7 \%$ et $55,8 \%$. On remarque ainsi, d'une part, que le pourcentage de fautes reconnues s'est stabilisé ces dernières années et, d'autre part, que l'écart entre les fautes admises et les fautes niées s'est réduit.

La prudence est de mise si l'on veut procéder à une interprétation de ces chiffres. S'agissant tout d'abord du nombre de cas traités annuellement par le Bureau d'expertises (qui pour 2012 est inférieur à 2011 et 2010), il suffit qu'un petit nombre de cas soit terminé juste à la fin de l'année précédente ou au début de l'année suivante pour modifier sensiblement ce nombre.

Le nombre total de 64 expertises terminées en 2012 reflète uniquement l'activité du Bureau d'expertises de la FMH et n'est que peu représentatif de la situation en matière de responsabilité civile dans les hôpitaux et chez les médecins en Suisse. Sans avoir de chiffres à disposition, nous savons que de nombreuses expertises privées sont effectuées chaque année et qu'un grand hôpital cantonal non universitaire est confronté, à lui seul, à environ vingt à trente cas de responsabilité civile chaque année.

Cette statistique montre donc uniquement combien d'expertises ont été établies dans les diverses disciplines par le Bureau d'expertises de la FMH et dans combien d'entre elles une faute de diagnostic et/ou de traitement a été constatée ou niée. Le petit nombre de données à disposition et le manque de valeurs comparatives ne permettent pas d'en tirer d'autres conclusions. On ne saurait donc, par exemple, prendre cette statistique comme base de calcul pour établir le pourcentage de fautes par discipline médicale ou de manière générale en Suisse. chaque complication ou attente de guérison déçue ne peuvent pas conduire à une expertise.

\section{Lien de causalité entre la faute et le dommage à la santé}

La réponse à la question de savoir si une faute a été commise dans le diagnostic ou le traitement ne clôt pas encore l'expertise. Si une faute a effectivement été constatée, il convient alors de déterminer si elle est aussi à l'origine du dommage à la santé évoqué par le patient. En effet, en matière de responsabilité civile, les conditions pour que le patient obtienne une réparation sont l'existence d'une faute (violation de l'obligation de diligence de la part du médecin), d'un dommage et d'un lien de causalité entre la faute et le dommage. Pour savoir s'il y a un lien de causalité, l'expert doit déterminer quel aurait été l'état de santé du patient si la faute n'avait pas été commise. Dans l'hypothèse où l'état de santé aurait été le même (soit si le dommage se serait également produit), la faute n'est pas causale.

Les cas dans lesquels les experts constatent une faute mais estiment que le lien de causalité est inexistant ou très peu probable sont relativement nombreux. Ainsi, en médecine comme ailleurs, chaque faute n'a - heureusement - pas forcément de conséquences graves ou négatives.

La statistique établie depuis des années ne recense pas ce critère de manière explicite. Pour 2012, le lien de causalité entre la faute constatée et le dommage a été reconnu clairement ou de manière très probable dans moins d'un quart $(21,8 \%)$ des dossiers concluant à l'existence d'une faute. Toutefois, dans le reste des cas avec fautes avérées, le lien de causalité a été nié ou considéré uniquement comme possible. Cela s'explique par le fait qu'il est souvent difficile de quantifier l'influence d'un seul facteur, en l'occurrence celui

\section{«Ainsi, en médecine comme ailleurs, chaque faute n'a - heureusement - pas forcément de conséquences graves ou négatives.»}

Enfin, la statistique ne montre pas le grand investissement en temps et en ressources engagé dans des demandes qui ne conduiront pas à une expertise, soit parce que la demande est incomplète ou alors parce que la société de discipline médicale concernée estime qu'il n'y a pas de matière suffisante pour organiser une expertise. Patients, avocats - de plus en plus nombreux -, médecins, assurances et autres institutions s'adressent, avec des questions multiples et variées, au Bureau d'expertises extrajudiciaires qui tente, dans la mesure du possible, de leur donner des informations utiles, même si les problèmes exposés n'entrent pas dans son domaine de compétence. Il est aussi souvent difficile de faire comprendre à un patient que la procédure est réglementée et que de la faute de diagnostic ou de traitement, sur le résultat global insatisfaisant. Il n'est pas rare que d'autres facteurs déterminants influencent le résultat, comme un pronostic de guérison préalablement défavorable dans tel cas particulier, des antécédents défavorables ou des maladies co-existantes.

\section{Information médicale au patient et communication entre médecin et patient}

La question de savoir si l'information médicale donnée au patient était suffisante ne peut pas à elle seule faire l'objet d'une expertise de la FMH. Elle peut toutefois être abordée parallèlement à la faute de diagnostic et/ou de traitement supposée si le patient fait valoir un défaut d'information. 
Il faut souligner ici combien il est important que cette information au patient soit suffisamment documentée. Il peut en effet arriver que les experts parviennent à la conclusion qu'aucune faute de diagnostic ou de traitement n'a été commise, mais que le médecin a violé son devoir d'information, soit parce qu'il a omis d'informer le patient soit parce que l'information était lacunaire ou non documentée. Dans ces cas, le fait qu'un risque se soit réalisé engage la responsabilité du médecin même si le traitement a été effectué avec diligence. Cela a été le cas à une reprise en 2012.

Il est également régulièrement apparu que la communication entre médecins et patients n'était pas optimale. Or, si les résultats du traitement ne correspondent pas entièrement aux attentes du patient ou si le traitement prend subitement une mauvaise tournure, une communication insuffisante de la part des médecins risque d'éveiller ou de renforcer chez les patients la présomption qu'une faute a été commise et leur ressentiment vis-à-vis du médecin.

\section{Assurance-qualité}

L'assurance-qualité revêt une grande importance dans la procédure suivie par le Bureau d'expertises, afin que les expertises puissent être le plus utile possible aux parties. Les démarches suivantes y contribuent:

- Les sociétés de discipline médicale proposent, pour chaque cas particulier, un/des expert/s. Le mandat est octroyé à ce/ces dernier/s au terme d'une procédure de récusation, une fois que les parties ont donné leur accord. Lorsque le cas l'exige, une équipe d'experts pluridisciplinaire est constituée. Le but visé est d'assurer une évaluation par des personnes neutres et compétentes en la matière, qui parlent si possible la langue du patient.

- Le schéma destiné aux experts, utilisé depuis des années, s'avère très utile car, en leur donnant une structure, il les aide à élaborer une expertise abordant tous les points déterminants, qui permette aux parties de régler le litige.

Sujets d'actualité du forum

Venez débattre avec nous! Dans la rubrique forum nous présentons régulièrement des sujets d'actualité politique, économique et scientifique ayant trait au système de santé. Donnez votre avis ou commentez les affirmations de vos confrères. Pour accéder au forum: www.bullmed.ch/forum/
- Un instrument particulier de l'assurance-qualité est la lecture du projet d'expertise par le service juridique de la FMH. En principe, les patients approuvent cette manière de procéder. La tâche des deux avocates est ainsi de soutenir les experts dans la rédaction de l'expertise, afin que cette dernière soit claire, complète, pertinente et, surtout, compréhensible pour des non-médecins.

\section{Formation des experts}

Les avocates du service juridique de la FMH participent régulièrement à des rencontres dont le but est de former les médecins à l'expertise médicale ou qui abordent la question de la responsabilité civile du médecin. Pendant l'exercice en revue, elles sont à nouveau intervenues lors des formations en exper- tises médicales de Swiss Insurance Medicine (SIM), à l'Institut de médecine légale à Zurich et lors du congrès annuel de la Société suisse de médecine interne (SSMI) à Bâle.

\section{Durée de la procédure}

La durée de la procédure est régulièrement critiquée, avant tout par les patients qui attendent une réponse prochaine à leurs questions. Il arrive parfois qu'une expertise puisse être close moins d'un an après l'envoi de la demande mais en principe, il faut compter avec un délai d'environ 17 à 18 mois à partir du moment où la demande est complète. Les raisons suivantes permettent, entre autres, d'expliquer ces longs délais: une procédure réglementée, transparente et acceptable par tous prend du temps; selon les cas, la recherche d'experts compétents dure à elle seule plusieurs mois, en particulier lorsque les experts proposés sont récusés par l'une des parties; à cela s'ajoute généralement le temps investi par le service juridique de la FMH pour lire les projets d'expertises, et le cas échéant, le temps dont a besoin l'expert pour réviser ou compléter son expertise (ce qui, en contrepartie, permet d'améliorer la compréhension et la pertinence de l'expertise); enfin, la recherche et l'obtention des documents nécessaires à la réalisation de l'expertise ne sont pas toujours aisées et les patients ne sont pas tous coopératifs (par exemple lorsque l'expert ne peut pas auditionner le patient parce que ce dernier se trouve à l'étranger pendant plusieurs semaines, cas qui n'est pas rare).

En outre, lorsque plusieurs experts ont été mandatés, chaque étape requiert plus de temps, depuis l'audition et l'examen du patient jusqu'à la rédaction finale de l'expertise. Il faut souligner que la charge de travail de nombreux experts est telle qu'ils trouvent à peine le temps nécessaire pour effectuer ce genre de mandats supplémentaires dans le délai souhaité; la plupart y sacrifient même une partie de leurs moments de loisir.

Le Bureau d'expertises extrajudiciaires offre une prestation, mais ne détient pas le monopole en matière d'établissement d'expertises. S'il accepte d'organiser une expertise, il veut pouvoir diriger la procédure conformément à son règlement et traiter toutes les parties équitablement.

\section{Conseil scientifique}

Le Conseil scientifique surveille l'activité du Bureau d'expertises extrajudiciaires sur mandat du Comité central de la FMH. Il n'a pas de compétence décisionnelle en ce qui concerne les différents cas traités mais décharge le Comité central de son devoir de surveillance et soutient le Bureau d'expertises en cas de difficultés lors d'une procédure d'expertise. Au cours de l'exercice en revue, le Conseil scientifique s'est réuni à deux reprises et a examiné par sondage 8 dossiers d'expertise et 2 décisions de non-entrée en matière. 
Le Conseil scientifique se compose des membres suivants: du Dr Bruno Lerf, président, du Dr Jürg Knessl et de $\mathrm{M}^{\mathrm{e}}$ Massimo Pergolis, avocat.

\section{Personnel}

Responsable du Bureau d'expertises extrajudiciaires, Susanne Friedli se charge des dossiers de la Suisse alémanique et du Tessin. Son suppléant, Sébastian Lerch, traite les dossiers de la Suisse romande.

A la fin du mois de septembre 2012, Lucia Rabia a quitté la FMH après 8 ans passés à son service. Durant ces 8 années, ellea supervisé le Bureau d'expertises avec volonté et engagement. Nous la remercions ici vivement pour sa précieuse collaboration et lui adressons nos meilleurs vœux pour la suite de sa carrière. Depuis le mois d'octobre 2012, le Bureau d'expertises extrajudiciaires est supervisé par Valérie Rothhardt, avocate, qui a été rejointe en novembre 2012 par Ursina Pally Hofmann, avocate et docteure en droit, toutes deux en fonction au service juridique de la FMH.

\section{Remerciements}

Pour pouvoir fonctionner, le Bureau d'expertises extrajudiciaires a besoin de la collaboration de nombreuses personnes. Nous remercions les sociétés de discipline médicale et leurs délégués pour leur précieux soutien ainsi que les experts pour leur disponibilité et le grand travail qu'ils accomplissent en vue d'éclaircir les cas. Le Bureau d'expertises remercie les médecins traitants ainsi que les directions d'hôpitaux qui, à la demande des patients, ont coopéré de manière ouverte et correcte à la réalisation d'expertises.
Susanne Friedli et Sébastian Lerch sont chargés du traitement des dossiers, depuis la réception de la première demande jusqu'à l'envoi des rapports d'expertise. Ils sont les interlocuteurs de toutes les parties et fournissent un grand travail de coordination et de conseil. Je leur adresse ici mes chaleureux remerciements pour leur engagement et leur motivation.

\section{Conseil aux patients}

Le Bureau d'expertises extrajudiciaires de la FMH propose aux patients, à leurs avocats et à d'autres conseillers de discuter du cas d'espèce par téléphone avec le collaborateur en charge du dossier avant le dépôt définitif de la demande.

Sur la base des recherches préliminaires, à quel moment et par quel médecin une faute auraitelle pu être commise? Quelles sont les autres causes de fautes possibles? En quoi pourrait consister le dommage à la santé? Quels sont les éléments particuliers que doit indiquer le Bureau d'expertises au délégué de la société de discipline médicale qui propose des experts? etc. Ces discussions préalables requièrent du temps, mais permettent d'éviter nombre de questions ultérieures et de faire avancer plus rapidement la procédure.

Les documents nécessaires au dépôt d'une demande d'expertise peuvent être obtenus auprès du Bureau d'expertises extrajudiciaires de la FMH, Case postale 6159, 3001 Berne, 03135912 10, fax $031 \quad 35912$ 12. D'autres informations peuvent être obtenues sous www.fmh.ch $\rightarrow$ Services $\rightarrow$ Bureau d'expertises extrajudiciaires. 